\title{
Tenossinovites De Quervain: uma nova proposta no tratamento cirúrgico
}

\author{
De Quervain's tenosynovitis: a new proposal of surgical treatment
}

\author{
William Albeiro Jimenez \\ $\mathrm{URIBE}^{1}$ \\ Gisela Del Pilar Puentes \\ BUENDIA $^{1}$ \\ Juan Manuel Florez \\ RODRIGUEZ ${ }^{1}$ \\ José de Gervais Cavalcante \\ VIEIRA FiLhO ${ }^{2}$
}

Trabalho realizado no Serviço de Cirurgia Plástica e Reparadora da Mão - Hospital Santa Casa da Misericórdia do Rio de Janeiro, Enfermaria 11, Rio de Janeiro, RJ, Brasil.

Artigo submetido no SGP (Sistema de Gestão de Publicações) da RBCP.

Artigo recebido: $27 / 4 / 2010$ Artigo aceito: $2 / 8 / 2010$

\begin{abstract}
RESUMO
Introdução: A tenossinovite estenosante De Quervain caracteriza-se por ser a inflamação da bainha do abdutor longo e extensor curto do polegar, no primeiro compartimento dorsal do punho, acometendo mais frequentemente as mulheres na faixa etária entre 30 e 50 anos. Essa doença está associada principalmente a trauma crônico secundário e sobrecarga das atividades diárias das mãos e punho, podendo também ser causada por outros fatores, mas em muitos casos não há uma causa bem definida. Objetivo: $O$ objetivo deste trabalho é apresentar nossa casuística de tenossinovite de De Quervain no Ambulatório de Cirurgia da Mão, os diferentes tratamentos e uma nova proposta cirúrgica utilizando um retalho fáscio-gorduroso de vizinhança. Resultados: Com a técnica proposta foram obtidos resultados satisfatórios, diminuindo as recidivas, com boa recuperação funcional e rápido retorno dos pacientes às atividades cotidianas.
\end{abstract}

Descritores: Mãos/cirurgia. Tenossinovites. Tenossinovite estenosante. Doença de De Quervain.

\section{SUMMARY}

Introduction: De Quervain's stenosing tenosynovitis it is characterized by being the inflammation of the hem of the long and extending abductor short of the thumb, in the $1^{\text {st }}$ number compartment of the fist, more frequently attacking the women in the age group between 30 and 50 years. That pathology is associated mainly to secondary chronic trauma and overload of the daily activities of the hands and fist, could also be caused by other factors, but in many cases no there is a very defined cause. Results:All the patients had a faster and satisfactory evolution with an amazing comeback to their quotidian activities.

Descriptors: Hand/surgery. Tenosynovitis. Tendon entrapment. De Quervain disease.

\section{INTRODUÇÃO}

Gradativamente vem crescendo o número de doenças osteomusculares relacionadas ao trabalho (DORT). Tais doenças vêm expandindo-se em todo o mundo. Mais especificamente, a tenossinovite de De Quervain caracteriza-se pela irritação ou tumefação dos tendões no lado do punho referente ao polegar. A inflamação espessa a bainha tendinosa e promove a constrição do tendão, em seu deslizamento na bainha. Isso pode causar um fenômeno de "disparo", em que o tendão parece travar ou "grudar", quando o paciente movimenta o polegar. Tipicamente, há envolvimento do primeiro compartimento extensor ${ }^{1}$.

A tenossinovite de De Quervain foi descoberta no final do século XIX, no ano de 1895, por um cirurgião suíço chamado Fritz de Quervain, sendo inicialmente chamada de tendinite de Quervain. Fritz de Quervain descreveu a entorse das lavadeiras, em função do desgaste sobre os tendões dos músculos abdutor longo do polegar e extensor

1. Médico residente do Serviço de Cirurgia Plástica e Reparadora da Mão - Hospital Santa Casa da Misericórdia do Rio de Janeiro, Enfermaria 11, Rio de Janeiro, RJ, Brasil.

2. Médico cirurgião plástico; Membro Titular da Sociedade Brasileira de Cirurgia Plástica; Chefe do Serviço de Cirurgia Plástica e Reparadora da Mão Hospital Santa Casa da Misericórdia do Rio de Janeiro, Enfermaria 11, Rio de Janeiro, RJ, Brasil. 
curto do polegar. Posteriormente, esta doença recebeu o seu nome, em sua homenagem ${ }^{2}$.

Esta doença é decorrente de espessamento do ligamento anular do carpo, no primeiro compartimento dorsal do punho. Sob a bainha sinovial trafegam os tendões dos músculos abdutor longo do polegar e extensor curto do polegar, que, com o tempo, apresentam um processo inflamatório local, que atinge os tecidos sinoviais peritendinosos e os tecidos próprios dos tendões. Sendo assim, a tenossinovite de De Quervain é uma das doenças mais frequentes que acomete o punho.

Este trabalho objetiva demonstrar a importância cirúrgica da relação entre o primeiro túnel extensor e a localização superficial do nervo cutâneo radial.

Além da técnica clássica, os autores apresentam sua experiência com a utilização de um retalho fáscio-gorduroso de vizinhança no tratamento cirúrgico de tenossinovite de De Quervain, buscando cumprir com os objetivos de tratar a doença, diminuir as recidivas e proporcionar cicatriz estética nos casos em que o tratamento conservador com o uso de corticoides locais não tem resposta satisfatória.

\section{MÉTODO}

No período de janeiro de 2008 a janeiro de 2009, 40 pacientes apresentavam tenossinovite de De Quervain no ambulatório. A idade dos pacientes variou de 25 a 65 anos (média de 45 anos). Em 34 (85\%) pacientes, a tenossinovite estava presente na mão dominante, em 6 (15\%), na mão não dominante (Tabelas 1 e 2$)$. Só dois ( $5 \%$ ) pacientes eram do sexo masculino e o restante do sexo feminino (95\%).

Os critérios de inclusão foram: dor em projeção do processo estiloide do rádio, com ou sem irradiação; perda da força; perda do movimento do polegar; e dor por meio da avaliação do teste de Finkelstein. Tal teste caracterizase pela flexão completa do polegar até a palma, seguido pelo desvio ulnar do punho; e o exame ultrassonográfico evidencia espessamento no primeiro túnel extensor.

Iniciado tratamento clínico com infiltrações com acetato de betametasona $2,71 \mathrm{mg}$ e fosfato sódico de betametasona $3 \mathrm{mg}$ (Celestone Soluspam ${ }^{\circledR}$ ) no primeiro túnel extensor e imobilização do polegar e punho, com avaliação médica em 3 semanas. Se o paciente apresentasse persistência dos sintomas, era feita uma segunda infiltração e nova avaliação 3 semanas após. Na persistência dos sintomas, era proposto o tratamento cirúrgico. $\mathrm{O}$ ato cirúrgico foi explicado detalhadamente aos pacientes.

No ato cirúrgico, foi utilizado bloqueio do plexo braquial a nível axilar e sedação. Foi realizado esvaziamento venoso e colocação de manguito pneumático graduado com o dobro da pressão arterial sistólica.

Realizou-se identificação do primeiro compartimento extensor com a presença da apófise do rádio, que foi confirmada na cirurgia com uma simples manobra, fazendo um desvio ulnar no punho e outra manobra fazendo hiperextensão do polegar. Foi realizada marcação em "S" itálico, próxima à apófise do rádio. Foi realizada incisão da pele e do tecido celular subcutâneo, localizando-se o nervo cutâneo radial, o qual é superficial. Foi realizada abertura do retináculo dos extensores (Figura 1), com a liberação dos tendões extensor curto do polegar e tendão abdutor longo do polegar (Figura 2). Foi buscada a presença do túnel acessório, e, em caso deste estar presente, foi realizada abertura do mesmo, sendo esta a técnica cirúrgica clássica.

A técnica proposta pelos autores consiste na confecção do retalho fáscio-gorduroso rabdomizado de vizinhança no subcutâneo (Figura 3), com dimensões de 2,5-3,5 cm de comprimento e 1,5-2,0 cm de largura, para fechamento e proteção do nervo cutâneo radial, sendo fixado com dois pontos simples separados, com nylon 4-0 (Figura 4). Foi graduada a sutura com o polegar em abdução, extensão e punho neutro. Após liberação do manguito, foi realizada hemostasia rigorosa, e fechamento de pele com fio de nylon 5-0.

Foi realizado curativo oclusivo dorso-radial, com abdução do polegar por duas semanas. Exercícios passivos de flexão do polegar foram encorajados após 24 horas de cirurgia.

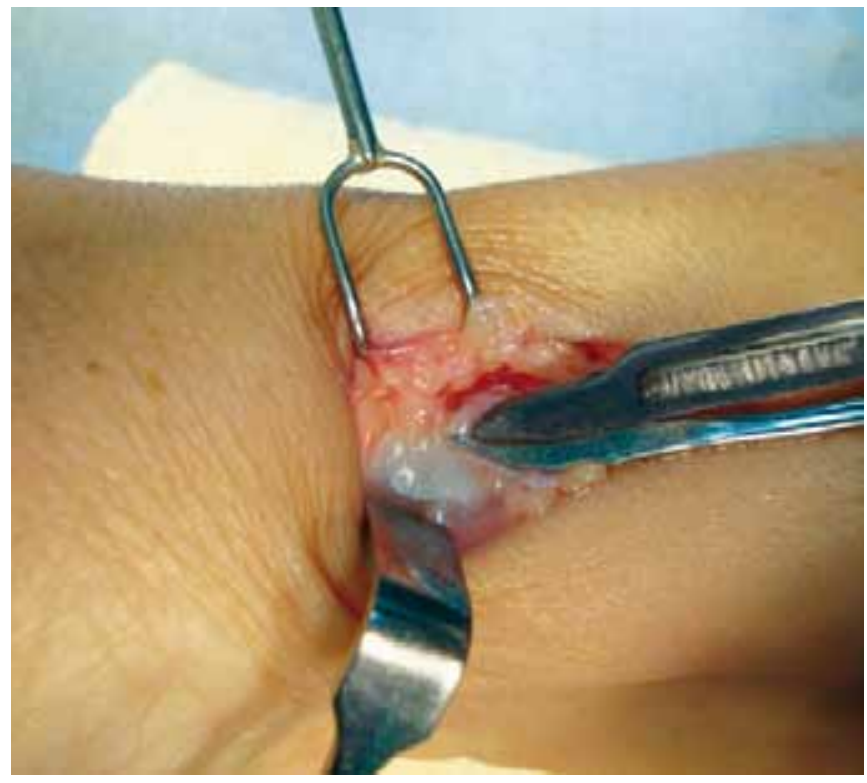

Figura 1 - Abertura do retináculo dos extensores. 


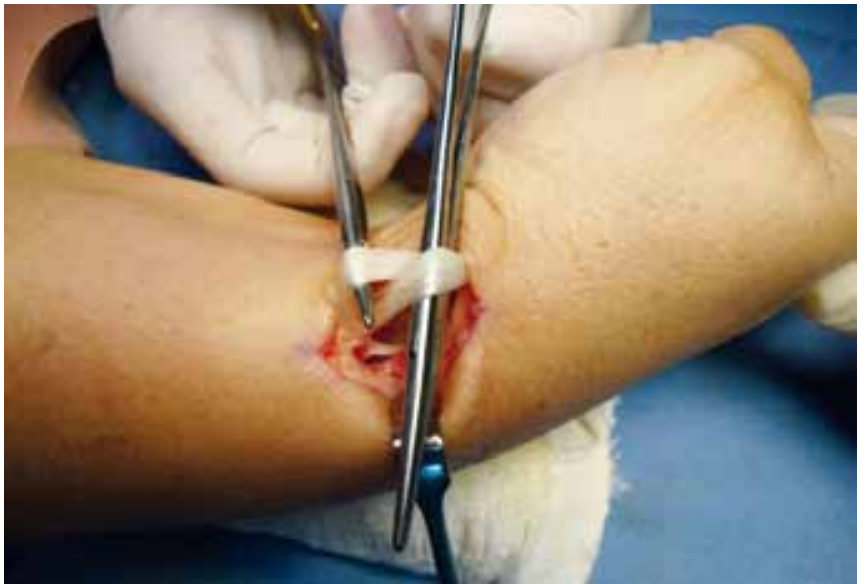

Figura 2 - Liberação dos tendões extensor curto do polegar e abdutor longo do polegar.

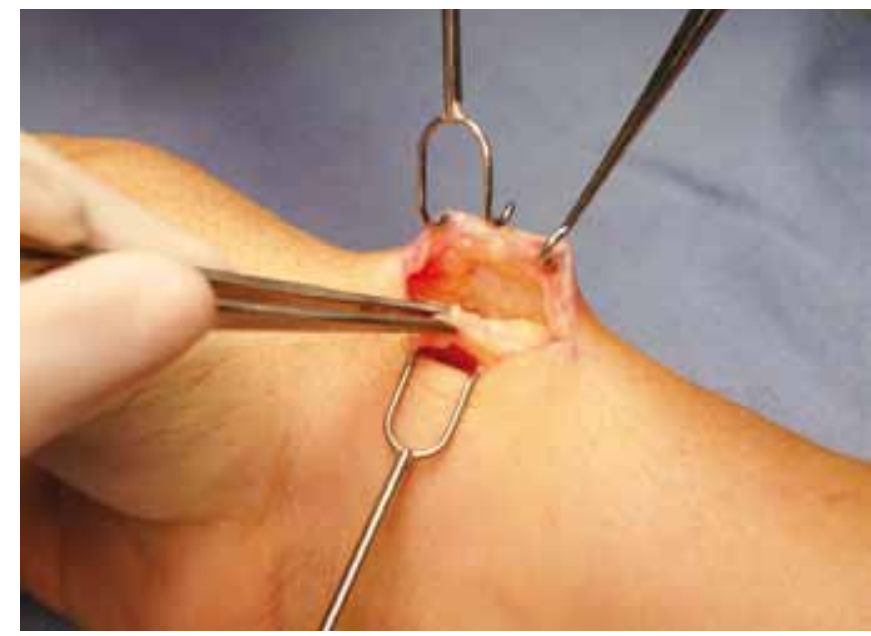

Figura 3 - Confecção do retalho fáscio-gorduroso da vizinhança.

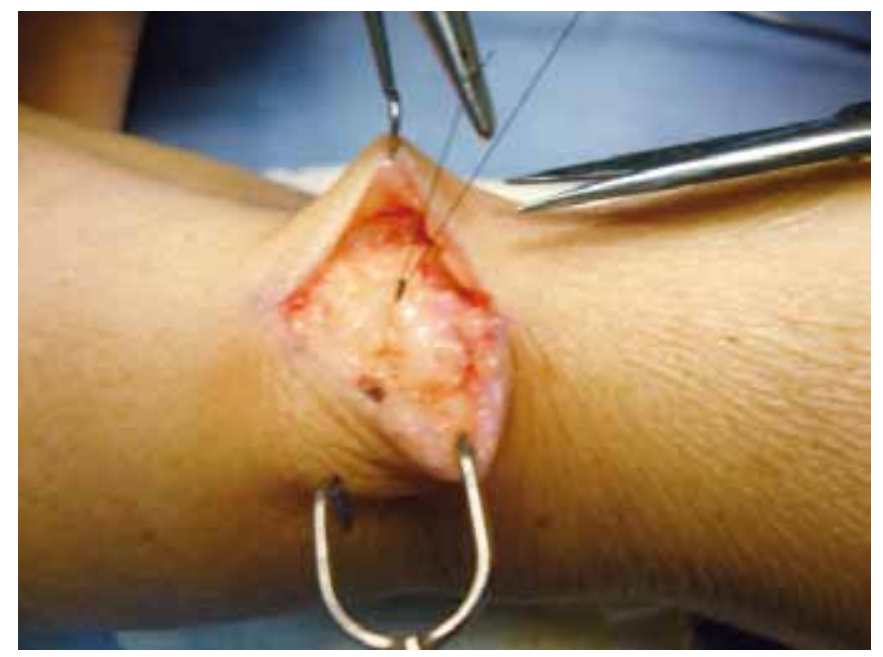

Figura 4 - Fixação do retalho com pontos simples.
Foram aplicados $2 \mathrm{~g}$ de cefazolina intravenosa peroperatória, permanecendo apenas analgesia pós-operatória. Todos os pontos foram removidos com duas semanas e os pacientes liberados para início da fisioterapia.

\section{RESULTADOS}

Dentre os 40 pacientes que apresentaram diagnóstico de tenossinovite de De Quervain no ambulatório, todos $(100 \%)$ foram submetidos a tratamento médico com infiltrações com corticoides. Doze (30\%) pacientes apresentaram melhora da sintomatologia, confirmada pelo teste de Finkelstein, aos $28(70 \%)$ restantes foi proposto o tratamento cirúrgico, que foi recusado por 2.

Vinte e seis $(65 \%)$ pacientes foram submetidos à cirurgia; $12(46,1 \%)$, só a abertura do retináculo do primeiro túnel extensor, com verificação de não terem um túnel acessório; 14 (53,9\%) utilizando o retalho fásciogorduroso de vizinhança, sendo escolhidos ao azar.

Realizada nova avaliação após de fisioterapia (10 seções) no grupo que foi submetido à cirurgia clássica; 3 (25\%) pacientes apresentaram hiperestesias da cirurgia, 2 $(16,6 \%)$, equimoses no local, que se resolveu espontaneamente; e $3(25 \%)$ apresentaram fibrose cicatricial que se resolveu com fisioterapia.

No grupo em que foi empregado o retalho fáscio-gorduroso de vizinhança, $5(35,7 \%)$ pacientes apresentaram hiperestesias após a cirurgia, que melhorou espontaneamente em todos eles.

Após seis meses de pós-operatório, no grupo que foi submetido a cirurgia clássica, $2(16,6 \%)$ pacientes persistiram com dor na projeção do processo estiloide do rádio, sem irradiação, perda da força, perda do movimento do polegar e teste de Finkelstein positivo; sendo os critérios de recidiva e corroborando com a literatura.

\section{DISCUSSÃO}

A tenossinovite estenosante de De Quervain caracterizase por ser a inflamação da bainha do abdutor longo e extensor curto do polegar, no primeiro compartimento dorsal do punho, acometendo mais frequentemente as mulheres na faixa etária entre 30 e 50 anos $^{3-8}$. Essa doença está associada principalmente a sobrecarga das atividades diárias das mãos e punho, trauma crônico secundário, podendo também ser causada por outros fatores, mas em muitos casos não há uma causa bem definida. O diagnóstico é clínico, obtido por meio do teste de Finkelstein, que é patognomônico da doença.

$\mathrm{O}$ retalho fáscio-gorduroso de vizinhança no tratamento da tenossinovite de De Quervain resulta como uma 
alternativa nos casos em que o tratamento conservador com o uso de corticoides locais não apresentou uma resposta satisfatória, persistindo a sintomatologia clínica.

Este retalho representa opção valiosa de prover uma apropriada cobertura de tecido, tendões e nervos, além de tratar a doença e proporcionar cicatriz reduzida e estética (Figuras 5 e 6).

Muitos tipos de retalhos faciais têm sido utilizados no tratamento de doenças da mão, tais como retalho de antebraço com um pedículo proximal, retalho fascial no qual o mesmo tem sido utilizado para envolver o nervo e minimizar a aderência depois do tratamento de neurólises. Neste tipo de retalho tem sacrifício da artéria radial.

Autores como Wilson et al. ${ }^{9}$ reportaram o uso do retalho fascial no antebraço para o tratamento de tenossinovite de De Quervain recorrente. Esses autores empregam um retalho da região radial distal do antebraço, onde utilizam um retângulo de tecido gorduroso de quatro centímetros de largura, criando um tubo no qual se cobre os tendões, proporcionando uma camada não aderente, a qual limita a fibrose pós-operatória. A camada contém vasos suficientes baseados em perfurantes distais da artéria radial, a qual fica intacta ${ }^{9}$. O retalho é girado a $180^{\circ}$ para alcançar o primeiro compartimento, esta técnica tem apresentado melhora na sintomatologia dos pacientes com doença de De Quervain recorrente, resultando em uma cicatriz maior.

Nossa proposta é feita para o tratamento cirúrgico inicial da doença do primeiro túnel com um retalho fáscio-gorduroso de vizinhança da fáscia dos extensores, que proporciona boa cobertura dos tendões e proteção do nervo sensitivo radial, evitando aderência e cicatriz maior.

\section{CONCLUSÃO}

A tenossinovite de De Quervain é uma doença limitante a todos os pacientes, impedindo-os de realizar suas atividades laborais e do dia-a-dia. Nosso retalho fáscio-gorduroso de vizinhança é uma excelente escolha para o tratamento da tenossinovite de De Quervain, já que fazemos uma liberação completa do retináculo dos tendões extensores do primeiro túnel, deixando-os livres e revisando a presença de túnel acessório. Nosso retalho cobre o nervo cutâneo radial, diminuindo assim o risco de recidiva e de outras complicações, tendo uma recuperação rápida e bem sucedida. A cirurgia é facilmente realizada em comparação à técnica do retalho facial baseado na artéria radial, que é mais complexa, deixando uma cicatriz maior na região do antebraço e com risco de necrose, pois é um retalho vascularizado.

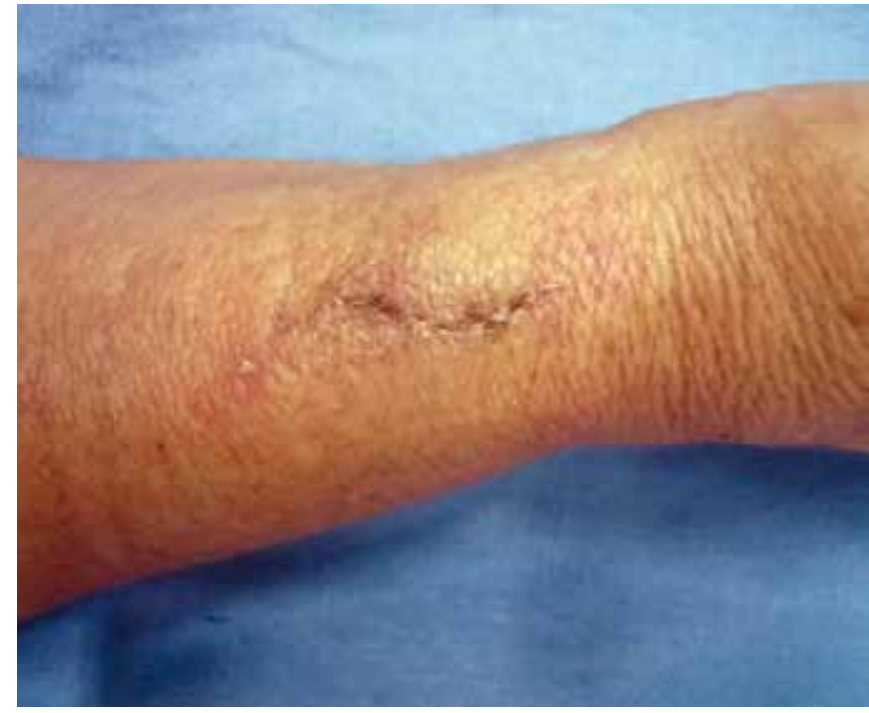

Figura 5 - Cicatriz de 3 semanas pós-operatório.

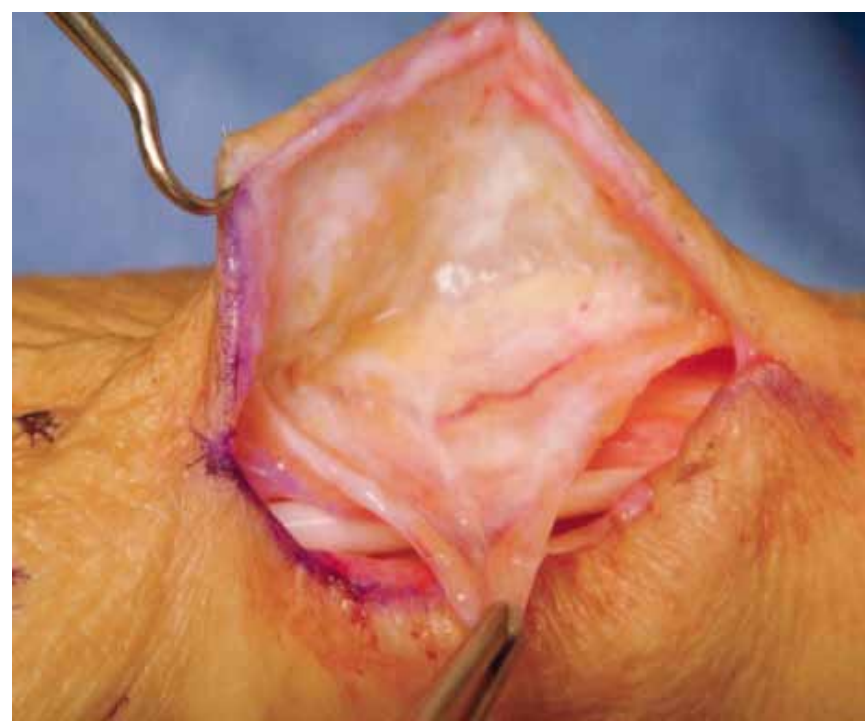

Figura 6 - Evidencia-se a cobertura das estruturas pelo retalho.

A técnica proposta resulta em uma cicatriz pequena, com um resultado mais estético e corrigindo o problema funcional sem apresentar recidiva depois de um período de pós-operatório considerável.

\section{REFERÊNCIAS}

1. Snider RK. Tratamento das doenças do sistema musculoesquelético. $1^{\mathrm{a}}$ ed. São Paulo:Editora Manole;2000.

2. Cherem AJ. A prevenção do pathos: uma proposta de protocolo para diagnóstico dos distúrbios osteomusculares relacionados ao trabalho. 
Disponível: www.eps.ufsc.br/disserta98/cherem/cap2.htm.

3. Clarke MT, Lyall HA, Grant JW, Matthewson MH. The histopathology of De Quervain's disease. J Hand Surg Br. 1998;23(6):732-4.

4. Harvey FJ, Harvey PM, Horsley MW. De Quervain's disease: surgical or nonsurgical treatment. J Hand Surg Am. 1990;15(1):83-7.

5. Kaneko S, Takasaki H, May S. Application of mechanical diagnosis and therapy to a patient diagnosed with De Quervain's disease: a case study. J Hand Ther. 2009;22(3):278-83.
6. Weiss AP, Akelman E, Tabatabai M. Treatment of De Quervain's disease. J Hand Surg Am. 1994;19(4):595-8.

7. Robinson RA. De Quervain's disease. J Chronic Dis. 1956;3(5):563-5.

8. Zingas C, Failla JM, Van Holsbeeck M. Injection accuracy and clinical relief of De Quervain's tendinitis. J Hand Surg Am. 1998;23(1):89-96.

9. Wilson IF, Schubert W, Benjamin CI. The distally based radial forearm fascia-fat flap for treatment of recurrent De Quervain's tendonitis. J Hand Surg Am. 2001;26(3):506-9.

\section{Correspondência para:}

William Albeiro Jimenez Uribe

Rua Teixeira de Melo, 50, 603 - Ipanema - Rio de Janeiro, RJ, Brasil - CEP 22410-010

E-mail:williamjimenezuribe@hotmail.com 